\title{
Politische Theorie und Ideengeschichte im Wissenschaftssystem
}

\author{
Peter Niesen (D) Armin Schäfer $(\mathbb{D}) \cdot$ Michael Zürn (D) - Wolfgang Knöbl • \\ Claudia Landwehr $\mathbb{D} \cdot$ Regina Kreide $\cdot$ Eva Marlene Hausteiner
}

Online publiziert: 27. Oktober 2020

(C) Der/die Autor(en) 2020

P. Niesen $(\bowtie)$

Fachgebiet Politikwissenschaft, Universität Hamburg, Allende-Platz 1, 20146 Hamburg, Deutschland E-Mail: Peter.Niesen@uni-hamburg.de

\section{A. Schäfer}

Westfälische Wilhelms-Universität Münster, Münster, Deutschland

E-Mail: aschaefer@uni-muenster.de

\section{Zürn}

Wissenschaftszentrum Berlin für Sozialforschung, Berlin, Deutschland

E-Mail: michael.zuern@wzb.eu

W. Knöbl

Hamburger Institut für Sozialforschung, Hamburg, Deutschland

E-Mail: direktion@his-online.de

C. Landwehr

Johannes Gutenberg-Universität Mainz, Mainz, Deutschland

E-Mail: landwehr@politik.uni-mainz.de

R. Kreide

Justus-Liebig-Universität Gießen, Gießen, Deutschland

E-Mail: regina.kreide@sowi.uni-giessen.de

E. M. Hausteiner

Rheinische Friedrich-Wilhelms-Universität Bonn, Bonn, Deutschland

E-Mail: evahausteiner@uni-bonn.de 


\section{Einleitung}

\section{Peter Niesen}

Politische Theorie und Ideengeschichte bilden historisch einen zentralen Pfeiler der Politikwissenschaft. Seit der Gründung der Disziplin in der Bundesrepublik sind sie fest in den Curricula verankert ${ }^{1}$ und waren bis an die Jahrhundertwende in allen modernen sozialwissenschaftlichen Instituten vertreten. Die Konsolidierung der Politikwissenschaft im Nachkriegsdeutschland entlang der Leitlinien der $1961 \mathrm{im}$ Auftrag der Deutschen Forschungsgemeinschaft verfassten „Denkschrift zur Lage der Soziologie und der Politischen Wissenschaft" erfolgte über die fachliche und personelle Ausdifferenzierung in drei Bereiche: Politische Theorie und Ideengeschichte, Innenpolitik und Vergleichende Regierungslehre sowie Internationale Politik (Bleek 2001, S. 310-312). Doch während die beiden empirischen Teilbereiche schnell Aufwuchs erfuhren, stagnierte der theoretische Anteil. Selbst Institute mittlerer Größe führen heute Diskussionen darüber, ob sie Politische Theorie und Ideengeschichte weiterhin zu ihrem Kerngeschäft zählen wollen.

Sie folgen damit einem Modell der Homogenisierung der Politikwissenschaft, das vor einer Generation an ansonsten angesehenen Instituten wie dem an der Universität Mannheim begonnen wurde, wo im Zuge einer Konzentration auf fachliche Stärken in der quantitativen Sozialforschung die verbleibende geisteswissenschaftlich angelegte Professur bei ansonsten starkem Aufwuchs gestrichen wurde (aus studentischer Perspektive vgl. Weber 2019). An der FU Berlin wurde zugunsten der Stärkung der Internationalen Beziehungen eine zentrale Theorieprofessur gestrichen. Anderswo wurden freiwerdende Professuren umgewidmet oder hybrid ausgeschrieben - für politische Theorie und/oder eine empirische Subdisziplin, wie in Stuttgart, Trier oder Leipzig (Ellersiek 2019), oder unter Absehung von Ideengeschichte und normativer Theorie gleich im Paradigma der rationalen Wahl angesiedelt, wie in Köln. Dort, wo Clusterausschreibungen mehrere politikwissenschaftliche Professuren gleichzeitig auf den Markt warfen, ist nicht selten eine Theorie-Stelle dabei, die im Unterschied zu den anderen nicht als Dauerstelle ausgeschrieben ist, wie an der TU München oder an der Viadrina Frankfurt/Oder.

Der Einfluss der Drittmittelfinanzierung spielt eine weitere Rolle, und zwar nicht aus dem realitätsfremden Grund, dass Theorieprofessorinnen keine Anträge stellten. ${ }^{2}$ Hier hat sich in den letzten 15 Jahren eine nahezu völlige Normalisierung der wechselseitigen Erwartungen eingestellt. Dennoch scheint der republikweite Trend zur Einwerbung von aus Drittmitteln anfinanzierten Professuren dazu zu führen, dass die Außenfinanzierung inhaltlich in Führung geht. Dies ist z. B. in Bezug auf BMBFProgramme zu beobachten, die innerhalb erfolgreicher Institute eine Reallokation

\footnotetext{
1 Vgl. das immer noch maßgebliche, 2003 von der DVPW entwickelte Kerncurriculum für die B.A.-Studiengänge der Politikwissenschaft, dessen fünf Kernbereiche Politische Theorie, Methoden, Politisches System der BRD, Vergleichende Politikwissenschaft und Internationale Beziehungen sind. https://www. dvpw.de/wir/bama/ (15.07.2020).

2 Die Beiträge dieser Debatte verwenden das generische Femininum, um auf alle Geschlechter zu verweisen.
} 
von Stellen an Spezialistinnen nach sich ziehen, die sich an einer politisch privilegierten Gegenwartsherausforderung profiliert haben. Die Policy-Nähe fremdfinanzierter Neuausschreibungen belohnt typische Qualifikationsprofile in Vergleichender Politikwissenschaft und Internationalen Beziehungen und verfehlt die oft umfassenderen, aber weniger anwendungsbezogenen Profile von Theoretikerinnen. Am Horizont zeichnet sich darüber hinaus eine Wende zum Department-Modell ab, die aus meritokratischer Sicht zu begrüßen ist - womöglich aber den Herdeneffekt hin zu einer Marginalisierung der Theorie nur verstärken könnte.

Die klassische Kopplung zwischen Theorie und Ideengeschichte scheint vielerorts gebrochen. Damit droht in politikwissenschaftlichen Studiengängen eine weitreichende Entprofessionalisierung, indem politiktheoretische und ideenhistorische Module ohne Forschungsanbindung gelehrt werden. Die Ausbildung der Lehrämter, in der die Geschichte des politischen Denkens ebenso zentral ist wie die zeitgenössischen Herausforderungen der Demokratie, würde entkernt. Gleichzeitig ist allerdings festzustellen, dass die Politikwissenschaft auf normative Stellungnahmen nicht vollends verzichten will. Im Gegensatz zur Enthaltsamkeit an Werturteilen, die ein positivistisches Wissenschaftsverständnis früher mit sich brachte, ist heute eine neue Lust an normativer Relevanz zu beobachten, die in den empirischen Teildisziplinen um sich zu greifen scheint. Normative Phänomene wie gesellschaftliche Desintegration, Ungleichheit, Bedrohungen der Demokratie und die Erosion politischer Legitimität werden aber häufig abgekoppelt von der historischen und begrifflichen Reflexion ihrer Grundbegriffe erörtert, freihändig verwendet und so gegen Kritik im eigenen Haus immunisiert. Dass Grundlagendiskussionen darüber ausbleiben, wann politikwissenschaftliche Modelle eigentlich angemessen und begründet angewendet werden, ist allerdings nicht nur den empirischen Teildisziplinen vorzuwerfen - ein Paradigmenstreit der Teildisziplinen wird zumindest nicht offen, und nicht vor breitem Publikum, ausgetragen.

Selbst die zunehmend erfolgreiche internationale Einbindung und Anerkennung der Politischen Theorie und Ideengeschichte in Deutschland, die vor einer Generation noch wenig ausschlaggebend schien, könnte geeignet sein, das Problem auf riskante Weise zu überdecken. Während die Lage der Politischen Theorie im englischsprachigen Ausland ebenfalls nicht ideal und den üblichen Budgetkämpfen unterworfen scheint, ist sie gerade an den besten Universitäten wohldefiniert und prestigeträchtig. Ihre Vertreterinnen hierzulande sind verurteilt, sich als Fachleute in einem ausdifferenzierten Bereich zu positionieren, den eher Political Theory und das Journal of Political Philosophy abstecken als APSR (American Political Science Review) oder die PVS (Politische Vierteljahresschrift). Sie stehen weltweit mitten in einer Epoche normalwissenschaftlicher Konsolidierung und der Kanonisierung moderner Klassiker, die nicht nur durch das Entstehen von Handbüchern zu produktiven Strömungen und großen Figuren (Jenco et al. 2020; Allen und Mendieta 2019), sondern ebenso sehr durch methodische Abrechnungen mit ihnen ins Bewusstsein treten (Forrester 2019). Vonseiten der restlichen Politikwissenschaft werden den verbleibenden Exemplaren ihrer Zunft große Freiheitsgrade eingeräumt: Ein Policing der Teildisziplin, wie Simone Chambers es einmal ausgedrückt hat, findet nicht statt. Doch womöglich bezahlt die Politische Theorie und Ideengeschichte ihre erfolgrei- 
che Autonomisierung mit einem zunehmend ungebundenen, weniger einflussreichen, weniger zentralen Charakter für das Fach Politikwissenschaft.

In dieser Situation ist es notwendig, die Frage aufzuwerfen, in welcher Fachidentität die Politische Theorie und Ideengeschichte zukunftsfähig sein wird. Wo gibt es Verbündete, wenn nicht in den eigenen Instituten? Verläuft der Anspruch auf Unentbehrlichkeit über die Außenwahrnehmung, in der kulturellen und politischen Öffentlichkeit und in der Beratungsfunktion, die die Theorie übernehmen kann? Sollte die Politische Theorie und Ideengeschichte die Eigenlogik eines emphatischen Theoriebegriffs betonen oder lieber ihre Gesprächsfähigkeit mit den anderen Teilbereichen der Politikwissenschaft akzentuieren? Die hier geführte Diskussion geht auf eine von der Fritz Thyssen-Stiftung geförderte Hamburger Sektionstagung zurück. Der Tagungsplanung lag die Vermutung zugrunde, dass es entscheidend sein wird, die Anschlussfähigkeit aus den umliegenden Subdisziplinen heraus und in sie hinein zu intensivieren, wie es auf den folgenden Seiten führende Vertreter der Vergleichenden Politikwissenschaft (Armin Schäfer), der Internationalen Beziehungen (Michael Zürn) und der Soziologie (Wolfgang Knöbl) anmahnen. Auf die Beiträge der Nachbarwissenschaftler antworten ganz unterschiedlich positionierte Vertreterinnen der Politischen Theorie und Ideengeschichte, die sich für die politikwissenschaftliche Professionalisierung und stärkere Integration (Claudia Landwehr), die Emanzipation von zu starren disziplinären Festschreibungen (Regina Kreide) oder für eine historisch fundierte, methodisch heterogene, aber öffentlichkeitswirksamere Auslegung aussprechen (Eva Marlene Hausteiner). Die Diskussion ist damit eröffnet; es ist nicht zu erwarten, dass sie damit beendet sein wird.

\section{Politische Theorie und Ideengeschichte aus der Perspektive der Vergleichenden Politikwissenschaft}

\section{Armin Schäfer}

Die Aufgabenstellung, über die Stellung der Politischen Theorie und Ideengeschichte im Wissenschaftssystem nachzudenken, ist nicht ohne Schwierigkeiten. Zwei Dinge wären mir leichter gefallen: Das Erste wäre gewesen, ein Loblied anzustimmen und zu betonen, wie sehr wir Politikwissenschaftlerinnen diese Subdisziplin brauchen, wie sehr empirische Forschung angewiesen auf normative Reflexion ist und wie sehr ich in meiner eigenen Arbeiten Anleihen bei der Politischen Theorie gemacht habe. Das hätte ich zwar alles ausführlich erörtern können, aber das war nicht der von den Organisatorinnen erwünschte Beitrag.

Eine zweite Möglichkeit wäre gewesen, Kritik an der empirischen Politikwissenschaft zu üben, an einer teilweisen szientistischen Engführung sowie an der Orientierung an den Wirtschaftswissenschaften in Teilen des Faches. Gerade vor Kurzem habe ich eine Präsentation erlebt, wo das Einzige, was gezeigt wurde, eine Formel war. Aber auch das war nicht mein Auftrag.

Stattdessen lautete die Anforderung, aus dem Blickwinkel einer Nachbardisziplin, der Vergleichenden Politikwissenschaft, etwas über den Zustand der Politischen Theorie zu sagen, ob sie unter Druck steht und worin dieser bestehen könnte. Ich soll 
mich zu den Spannungen äußern zwischen der eher empirisch-positivistischen Vergleichenden Politikwissenschaft und der Politischen Theorie und Ideengeschichte. Drei Punkte möchte ich andiskutieren.

I Die Vergleichende Politikwissenschaft orientiert sich am angloamerikanischen Diskurs, und Internationalisierung gehört zum eigenen Selbstverständnis dazu. Ich glaube, dass man inzwischen - wenn man einen Ruf auf einen Lehrstuhl für Vergleichende Politikwissenschaft erhalten möchte - Veröffentlichungen in angesehenen englischsprachigen Fachzeitschriften braucht. Und genauso bedeutend ist, dass man an internationalen Konferenzen teilnimmt. Wenn ich dort bin, habe ich den Eindruck, nicht allzu viele Mitglieder der Sektion Politische Theorie und Ideengeschichte anzutreffen. Nun kann es natürlich sein, dass ich die falschen Konferenzen oder die falschen Panels besuche, aber es könnte auch sein, dass es unterschiedliche Anforderungsprofile zwischen Subdisziplinen gibt. In vielen Teilbereichen der Politikwissenschaft ist es völlig selbstverständlich, zu einer internationalen - das heißt englischsprachigen - Fachöffentlichkeit zu sprechen. Und mein Eindruck ist, dass dies in der Politischen Theorie und Ideengeschichte weniger selbstverständlich ist. Damit hängt zusammen: Wo publiziert man? Wie publiziert man? In weiten Teilen der Politikwissenschaft ist es selbstverständlich, auch auf Englisch zu publizieren. Ich selbst habe zwar immer wieder auf Deutsch geschrieben, weil ich denke, es ist auch Teil unserer Aufgabe, sich an die Öffentlichkeit zu richten, aber auf Englisch zu publizieren und sich dem Peer-Review-Prozess zu unterziehen, ist unumgänglich. In der Politischen Theorie und Ideengeschichte, so mein Eindruck, zielt man weniger auf die englischsprechende Fachöffentlichkeit. Als Begründung hierfür höre ich immer wieder, das sei der Fall, weil man sich in der Fremdsprache nicht genauso präzise ausdrücken könne und weil bestimmte Theoriebegriffe in anderen Sprachen nicht in derselben Form zur Verfügung stünden. Das mag zum Teil stimmen, aber je mehr Kolleginnen auch auf Englisch veröffentlichen, desto mehr werden sich bestimmte Begriffe durchsetzen.

II Die Politikwissenschaft wird insgesamt, glaube ich, wissenschaftlich strenger. Sie ist stärker als früher methodenorientiert, und sowohl bei qualitativen als auch quantitativen Analysen steigen die Ansprüche. Insbesondere bei den quantitativen Methoden ist nicht immer alles leicht nachzuvollziehen, und ich muss zugeben, dass auch ich nicht jede Verästelung so ohne Weiteres verstehe. Aber es wird zu Recht verlangt, dass man mindestens die Beiträge in der American Political Science Review oder auch der Politischen Vierteljahresschrift verstehen können muss. Ich denke, dass wir in der Politikwissenschaft alle in der Lage sein müssen, empirische Aufsätze, die auch mit Regressionsanalysen oder Ähnlichem arbeiten, zu verstehen - vielleicht auch nur, um sie kritisieren zu können, aber diese Methodenkompetenz muss vorhanden sein.

In meiner Wahrnehmung besteht eine Kluft zwischen empirisch und theoretisch arbeitenden Kolleginnen, die nicht kleiner zu werden scheint. Vor einigen Jahren stellte ich bei einer Sektionstagung der Politischen Theorie eine empirische Studie zur Nichtwahl vor. Anschließend erhielt ich die Reaktion, dass das, was ich vorgestellt habe, in dieser Sektion nicht zugänglich gewesen sei. Die Mitglieder des 
Arbeitskreises „Wahlforschung und politische Einstellungen“ hatten jedoch, wenige Wochen zuvor, den Eindruck, mein Vorgehen sei nicht anspruchsvoll genug. Da öffnet sich eine Kluft, die Sprachbarrieren von beiden Seiten schafft. Wir sollten dafür sorgen, dass wir uns wechselseitig verstehen, und dazu gehört, denen, die sich mit Theorie auseinandersetzen, abzuverlangen, dass sie auch verstehen, was die Politikwissenschaft empirisch leistet - und idealerweise würden sich empirisch Forschende auch intensiver mit Theoriedebatten beschäftigen.

III Ein dritter Punkt betrifft meines Erachtens nach das Fach Politikwissenschaft insgesamt. Wie gut schaffen wir es, den öffentlichen Diskurs aufzuklären und zu wichtigen Fragen gehört zu werden? Wir schaffen das (noch) nicht in dem Maße, wie es sein sollte oder könnte. Es ließe sich ja fragen: Warum gibt es gerade einen Aufschwung der Politischen Theorie in den USA? Die Antwort könnte lauten, aufgrund der Menge drängender gesellschaftlicher Probleme, zu denen wir uns äußern sollten. Wem schulden wir Solidarität und wo verlaufen die Grenzen von Solidarität? Wie lässt sich Demokratie denken, wenn Öffentlichkeit digital fragmentiert wird? Wie sollten die Kosten verteilt werden, die anfallen, um den Klimawandel aufzuhalten oder zumindest zu verlangsamen beziehungsweise die Folgen zu bearbeiten? Und was wäre eine gerechte Art, damit umzugehen?

In der Politikwissenschaft wird dazu viel geforscht, und es gibt eine Menge kluger Positionen. Aber ich höre oder lese relativ selten, dass Beiträge aus der Sektion Politische Theorie und Ideengeschichte diesbezüglich rezipiert werden und es dadurch gelingt, den politischen Diskurs zu solchen und vielen anderen Themen aufzuklären. Sich mehr einzumischen, wäre aber wünschenswert, weil das Niveau der öffentlichen Debatte häufig nicht übertrieben anspruchsvoll ist. Bei der Diskussion um die Spitzenkandidatur in der EU war mein Eindruck, dass bei vielen Journalistinnen ein Grundverständnis fehlte, wie verschiedene Regierungssysteme funktionieren, aber offensichtlich ist es uns als Fach nicht gelungen, da aufklärend zu wirken. Mein Eindruck ist - um den Kontrast nochmals zu benennen -, dass es empirisch arbeitenden Politikwissenschaftlerinnen etwas leichter fällt, Gehör zu finden, weil sie etwas ,,anzubieten“ haben, was Medienschaffende mögen, nämlich mehr oder wenige präzise Zahlen oder eingängige Grafiken.

Wie lässt sich die öffentliche Wahrnehmung verbessern? Ich glaube, beispielsweise der theorieblog (https://www.theorieblog.de/) ist ein guter Ansatz. Die dortigen Diskussionen über wichtige neue Bücher sind ein guter Ansatz. Ich denke, wir brauchen mehr derartige Instrumente, um einer breiten Öffentlichkeit unsere Analysen zur Verfügung zu stellen, in der Hoffnung, dass die Erkenntnisse durchsickern und sich der Diskurs über einen längeren Zeitraum verändert.

Bei allen oben genannten Punkten sehe ich Veränderungsbedarf und würde sagen, es gibt möglicherweise ein Krisenmoment in der Politischen Theorie und Ideengeschichte. Ich benenne das deshalb, weil ich davon überzeugt bin, dass wir Empirikerinnen ein teilweise verkürztes Verständnis von Begriffen - auch Grundbegriffen - der Demokratie oder der Politikwissenschaft haben. Mit zwei Standardreferenzen auf die Demokratietheorie (nach dem Motto „Dahl geht immer“) bestehen wir die meisten Vorträge und können dann zur Empirie übergehen. Insofern müssen wir uns ernsthafter darum bemühen, dass es ein intensiveres Gespräch zwischen den Teil- 
disziplinen gibt, und ich habe die Hoffnung, dass Vergleichende Politikwissenschaft und Politische Theorie voneinander profitieren würden, wenn sie sich gegenseitig stärker zur Kenntnis nähmen und sich nicht nur oberflächlich mit dem Anderen auseinandersetzten.

\section{Politische Theorie aus der Perspektive der Internationalen Beziehungen}

\section{Michael Zürn}

Ich werde mich der Politischen Theorie im Wissenschaftssystem aus der Perspektive eines empirisch orientierten Politikwissenschaftlers mit theoretischen Neigungen aus dem Teilbereich der Internationalen Beziehungen widmen. Das, was ich beitragen möchte, beinhaltet letzten Endes beides: Lob und Kritik.

Zunächst möchte ich ganz bewusst die Bedeutung einer primär normativ verstandenen Politischen Theorie hervorheben. Politische Theorie verstehe ich dabei als Reflektion über die gute politische Ordnung und deren Begründung sowohl aus ideengeschichtlicher als auch aus begriffsanalytischer Perspektive. Dazu gehört selbstverständlich auch, dass die Kategorien, die dort verwendet werden, aus kritischer Perspektive hinterfragt werden. Die Politische Theorie unterscheidet sich damit von Theorien der Politik, die primär auf deskriptive und kausale Analysen real existierender politischer Systeme oder Politiken zielen. Im Folgenden geht es primär um die Politische Theorie. Und die ist - um es gleich in aller Deutlichkeit zu formulieren - meiner Meinung nach grundlegend und unverzichtbar für Theorien der Politik und schlechthin alle politikwissenschaftlichen Analysen.

Die Politische Theorie ist aus drei Gründen grundlegend und unverzichtbar. Erstens ist für mich Politikwissenschaft ohne Kritik unvorstellbar. Kritik braucht aber normative Referenzpunkte (Forst 2020). Kritik auf der Grundlage von normativen Referenzpunkten, die nicht reflektiert werden (und Reflektion ist ja der Kern der Aufgabe der Politischen Theorie), ist leer. Insofern wir Politikwissenschaft als eine - auch kritische - Wissenschaft verstehen, beinhaltet sie notwendigerweise einen kritischen Diskurs über die normativen Referenzpunkte der Gesellschaft und der Politikwissenschaft. Das ist die vornehme Aufgabe der Politischen Theorie.

Das zweite Argument für die Unverzichtbarkeit der Politischen Theorie beinhaltet eine Infragestellung der Selbstbeschreibung vieler meiner Kolleginnen aus den empirisch orientierten Teilbereichen. Deren dominante Selbstbeschreibung ist, dass die empirische, kausal orientierte Politikwissenschaft primär über Puzzles arbeitet, wobei Puzzles üblicherweise als die Differenz der Erwartungen einer Theorie und dem, was man beobachtet, definiert werden. Das sind die Puzzles, wie sie von der Wissenschaftstheorie anhand der Analyse der Naturwissenschaften - vor allem der Physik - als Treiber des wissenschaftlichen Fortschritts identifiziert worden sind (Kuhn 1962). Für die Gesellschaftswissenschaften und mithin die Politikwissenschaft scheint mir aber häufig eine andere Form des Puzzles erkenntnisleitend zu sein. Es geht oft um normative Puzzles, also um die Differenz zwischen dem, wie wir glauben, wie es sein soll und dem, wie es ist. Das ist eine der, wenn nicht sogar die zentrale Quelle(n) wichtiger Fragestellungen in der Politikwissenschaft. Ein 
geradezu banales Beispiel ist die Frage: „Warum Krieg?“. Die Relevanz der Frage ergibt sich nicht aus der Differenz der Beobachtung eines Krieges mit einer sozialwissenschaftlichen Theorie, die dauerhaften und kompletten Frieden erwarten lässt. Diese Frage konstituiert sich vielmehr aus der normativen Differenz zwischen Krieg und Frieden und der Tatsache, dass wir Krieg als Störung empfinden und deswegen wissen möchten, warum es eigentlich Kriege gibt. Das geht weiter mit der Frage „Warum können autoritäre politische Systeme stabil sein?“; ,Warum sind europäische Institutionen nur bedingt demokratisch?“; ,Wie kommen normativ unbegründbare Ungleichheiten zustande?“. Das sind nur einige Kernelemente des aktuellen Programms der empirischen Politikwissenschaft: Ungleichheit, Europäische Union, Kriege oder das Aufkommen autoritärer politischer Systeme. Sie alle entstammen weniger dem empirischen Puzzle - also der Differenz zwischen Prognose der Theorie und Beobachtung - als vielmehr der Differenz zwischen Sollen und Sein, also zwischen unseren normativen Erwartungen oder Wünschen und dem, was beobachtet wird. Das gilt übrigens auch für die Demokratieforschung, die nicht selten die Abweichung der Praxis von den Prinzipien durch Funktionsnotwendigkeiten erklärt. Die Bereitstellung der normativen Maßstäbe, vor deren Hintergrund erst normative Puzzles entstehen können, ist also der zweite Grund, warum die normative Politische Theorie aus meiner Sicht ein zwingender Bestandteil aller Politikwissenschaft ist.

Ein dritter Punkt ergibt sich aus der hochgradig institutionalistischen Orientierung weiter Teile der empirischen Politikwissenschaft. Diese muss sich aber hin und wieder von ihrem Fokus auf die Institutionen lösen, um die Effekte gesellschaftlichen Wandels erfassen und begreifen zu können. Um ein Beispiel zu nehmen: Innerhalb der Vergleichenden Politikwissenschaft wird auf die Frage ,Was zeichnet eine Demokratie aus?" sehr häufig eine institutionalistische Antwort gegeben, die sich an der parlamentarischen Demokratie orientiert und beispielsweise auf die Arbeiten von Robert Dahl (1989) verweist. Die zentralen Begriffe, die dann genannt werden, sind freie Wahlen, Parlamente, Parteienwettbewerb und Ähnliches mehr. Die dahinterstehenden normativen Prinzipien sind dabei aber nicht immer im Fokus der Betrachtungen. Was ist aber, wenn im Zeitalter der Globalisierung die sozialen und die politischen Räume tatsächlich auseinanderlaufen? Dann ist die einfache institutionelle Antwort nicht mehr ausreichend. Eine Entscheidung durch das in freien Wahlen konstituierte Parlament der Bundesrepublik Deutschland ist dann nicht mehr zwingend demokratischer als eine Entscheidung durch die Mitgliedsstaaten der WTO, die in Hinterzimmern vorbereitet worden ist (Held 1995; Zürn 2004). Das normative Prinzip, wonach alle diejenigen, die von einer Entscheidung betroffen sind, auch ein Mitspracherecht haben sollten, führt zu dem Urteil, dass Entscheidungen durch ein gewähltes Parlament mit territorialer Beschränkung an demokratischer Qualität verlieren, wenn sie weitreichende Externalitäten aufweisen. Durch die Inkongruenz von sozialen und politischen Räumen verlieren also unter Umständen territorial begrenzte demokratische Institutionen an demokratischer Qualität. Eine solche Erinnerung an die normativen Prinzipien, die Entscheidungen demokratisch machen, ist wiederum eine zentrale Aufgabe der Politischen Theorie. Das ist der dritte Punkt, warum die Politische Theorie unverzichtbar für die Politikwissenschaft als Ganzes ist: Sie muss die institutionalistischen und operationalen Abkürzungen 
der empirischen Politikwissenschaft hinterfragen und sich mithin in die empirische Forschung einmischen.

Mit dem Postulat der Einmischung in die empirische Forschung komme ich zum kritischen Teil meiner Anmerkungen. Die Gründe, die ich für die Bedeutung der Politischen Theorie angeführt habe, sagen vermutlich keiner Theoretikerin etwas wirklich Neues. Im Prinzip glauben die Vertreterinnen der Politischen Theorie alle an die Unverzichtbarkeit der normativen und kritischen Reflektion. Daraus speist sich auch ein hohes Maß an Identität mit einem klaren Verständnis, wer dazu gehört und wer nicht. Gleichzeitig meint die Politische Theorie, hierzulande nicht genügend Anerkennung von außen zu erfahren. Daraus entwickelt sich - so meine ich zu beobachten - eine ambivalente Grundhaltung. Einerseits wird das eigene Tun als unverzichtbar und schon auch „,höherwertig“ bzw. ,tieferschürfend“ angesehen, aber andererseits erfahre es nicht die Wertschätzung, die es eigentlich verdient hätte. Wir sind - so meine Wahrnehmung der Selbstwahrnehmung der Politischen Theorie diejenigen, die sich ungerechtfertigterweise in der Defensive finden und von der Mehrheit der Empirikerinnen z. B. bei Stellenausschreibungen und -besetzungen an den Rand gedrängt werden.

Daraus ergibt sich eine in der Politischen Theorie recht weit verbreitete Grundhaltung, die ich hier - als Diskussionsanstoß - kritisieren möchte. Es handelt sich um die Neigung, empirische Fragen nur mit spitzen Fingern anzufassen, weil das doch die alleinige Aufgabe der Heerscharen von empirischen Wissenschaftlerinnen ist. Das münzt sich in eine methodische Homogenität um, die mit einem leisen Überlegenheitsanspruch daherkommt. „Beobachten, Messen und Testen, das sollen die anderen machen, wir übernehmen das Denken." In der Diskussion mit Freundinnen aus der Politischen Theorie taucht dann der Satz auf: „Hmm, das ist jetzt eine empirische Frage, dazu kann ich nichts sagen ...."

Eine Subdisziplin bzw. eine Domain of Knowledge sollte sich aber nicht durch methodische Homogenität definieren. Daher sollte auch die Politische Theorie nicht methodisch durch Argumentations-, Ideen- und Begriffsanalyse bestimmt werden, sondern durch den Forschungsgegenstand: durch die Frage nach der guten politischen Ordnung. An diesen zentralen Forschungsgegenstand der Politischen Theorie kann man aber nicht nur normativ, sondern auch pragmatisch und empirisch herangehen.

Genau das ist der Wunsch, den ich an die Politische Theorie richten möchte: nämlich, dass sie sich unter Anerkennung der eigenen Fragestellung für pragmatische und empirische Forschung öffnet. Die pragmatische Dimension hat - so meine ich Armin Schäfer schon mit der Frage ,Was heißt denn eigentlich Gerechtigkeitstheorie in unserer Zeit?" angesprochen. Wenn wir heute beispielsweise über das Klima diskutieren, dann stellen sich unvermeidlicherweise Gerechtigkeitsfragen. Diese werden aber in der Öffentlichkeit teilweise frei von Sinn und vor allem ohne theoretische Grundierung diskutiert. Aus meiner Sicht ist das z. B. der Fall, wenn gerufen wird: „Klimapolitik ist sozial ungerecht.“ Mit dieser, gerade auch aus dem linken Spektrum vorgetragenen, Mahnung wird so getan, als ob der ungebremste Klimawandel verteilungsneutral erfolgen würde. Der fortschreitende Klimawandel wird aber ohne politische Eingriffe in seine distributiven Konsequenzen viel weitreichender sein als seine Bearbeitung. Die Politische Theorie - so mein Wunsch - sollte viel mehr Teil 
dieser Debatten sein als sie es derzeit ist. Dazu muss sich die Politische Theorie aber aus den Höhen der Begründung mehr in den Bereich der Anwendung herablassen, was natürlich auch empirische Forschung zum Bestandteil der Argumentation werden lässt. Bei derartigen pragmatischen Debatten hilft ein Satz wie „Nehmen wir mal an, eine Klimaerwärmung von $2{ }^{\circ} \mathrm{C}$ mache die Anschaffung von 20 Mio. Klimaanlagen notwendig“" nicht wirklich weiter.

Empirische Forschung sollte aber nicht nur Eingang in die Politische Theorie finden, sondern teilweise auch selbst von der Politischen Theorie durchgeführt werden. Denn viele der Debatten, die die Politische Theorie prägen, verweisen notwendigerweise auf unterschiedliche Hintergrundannahmen über die Funktionsweisen von Menschen und Gesellschaften. Mir erscheint die Selbstverständlichkeit problematisch, mit der dennoch bestimmte Annahmen über den Menschen und das Funktionieren von Gesellschaften in der Politischen Theorie aus dem Teilgebiet heraus verwiesen werden. Ein einfaches Beispiel: Die Frage, ob die Menschen hinter dem Schleier des Nichtwissens tatsächlich so agieren, wie Rawls das vermutet, scheint mir sehr plausibel, aber empirisch offen zu sein. Sie könnte etwa im Zuge experimenteller Forschungen leicht angegangen werden. Es scheint mir aber in der Politischen Theorie heutzutage nur wenige zu geben, die diese ,untersuchbaren Fragen“ bearbeiten. Das muss aber nicht so sein. Jürgen Habermas hat sich nie gescheut, empirische, in seinem Falle meist historische Untersuchungen in seine Argumentationen einfließen zu lassen. Die Frage, welche Ideen über das Funktionieren von Gesellschaft eigentlich hinter republikanischen und liberalen Demokratievorstellungen stehen, und was das wiederum heißt, also unter welchen Bedingungen republikanische und liberale Modelle überhaupt funktionieren können, ist aber etwas, was seitens der Politischen Theorie heute gern delegiert wird. Delegiert wird es meistens mit dem Unterton, dass für diese bestenfalls halbintellektuellen Aufgaben die bodenständigen Empirikerinnen zuständig sind.

Genau diese eigenartige Mischung aus gefühlter Überlegenheit und gefühlt mangelnder Wertschätzung übersetzt sich in eine innere Homogenität und auch einen bestimmten Habitus der Politischen Theorie. Es ist der Habitus der verkannten Avantgarde. Das ist die These, die ich zur Diskussion stellen möchte. Gleichzeitig möchte ich aber noch einmal mit großer Überzeugung zum Ausdruck bringen, dass ich die Politische Theorie für unverzichtbar halte und sie als absolut zentralen Bestandteil der Politikwissenschaft ansehe. Sie würde aber aus meiner Sicht noch bedeutsamer und unverzichtbarer werden, wenn sie ihre Domain of Knowledge nicht primär methodisch, sondern substanziell bestimmt. Ich fordere damit nicht, dass die Politische Theorie jetzt die Theorien der Politik entwickeln und testen sollte. Vielmehr geht es mir darum, dass die empirischen Aspekte, die den Problemstellungen der Politischen Theorie eigen sind, auch dort - und zwar gerne in Kooperation - bearbeitet werden und nicht einfach an die anderen Teildisziplinen wegdelegiert werden. Das würde die Diskussionskultur innerhalb des Fachs sowie die Rolle der Politischen Theorie stärken. Vielleicht können diejenigen aus der Politischen Theorie, die an dem Projekt der Internationalen Politischen Theorie beteiligt sind als Leitbild fungieren. 


\section{Soziologische Perspektiven auf die Politische Theorie und Ideengeschichte}

\section{Wolfgang Knöbl}

Folgt man dem jüngst publizierten Aufsatz von Reinhard Mehring (2019) mit dem Titel „Ironie der Theorie“, so spaltete sich die nach 1949 in der Bundesrepublik Deutschland betriebene Politische Theorie und Philosophie (und dies hatte dann auch Auswirkungen auf die Ideengeschichte) in zwei große Lager, eines, das mit dem Namen von Jürgen Habermas und dem von ihm vertretenen ethischen Idealismus und Normativismus verbunden ist, und - in kritischer Reaktion darauf - eines, für das die Bezeichnung politischer Realismus gefunden wurde, wie er prominent etwa von Herfried Münkler vertreten wird. ${ }^{3}$ Nimmt man dies zum Ausgangspunkt und fragt nach der Bereitschaft und Offenheit einer theoretisch ambitionierten Soziologie, auf diese beiden Angebote zuzugehen, so wird man jedenfalls mit Bezug auf normativistische Positionen von einer großen Zögerlichkeit und Zurückhaltung der Soziologie sprechen können. Man mag als ein Indiz für eine solche Behauptung auf die Tatsache verweisen, dass es der sogenannten Kritischen Theorie seit den frühen 1980er-Jahren immer weniger gelungen ist, von sich aus ernsthafte soziologische Beiträge oder Zeitdiagnosen zu liefern; das noch im alten Frankfurter Institut erkennbare Streben nach einer engen Klammer zwischen Philosophie und gesellschaftswissenschaftlichen Disziplinen rückte immer weiter in den Hintergrund. Angeboten wurde aus Frankfurt im Wesentlichen und seit den 1990er-Jahren fast ausschließlich Sozialphilosophie, Politische Philosophie und Rechtstheorie. Nach der Publikation der Theorie des kommunikativen Handelns (1981) war Habermas selbst, aber auch diejenigen, die wie Axel Honneth oder Rainer Forst seinen Spuren folgten, zunehmend weniger an systematischer soziologischer Theoriearbeit interessiert. Kein Wunder, dass dann aus der Soziologie heraus die Anschlussfähigkeit und -willigkeit des Frankfurter Normativismus immer skeptischer beurteilt wurde! All dies ist den Frankfurtern (und all denjenigen, die mit ihnen kooperierten) ganz sicherlich nicht allein zum Vorwurf zu machen. Vielmehr ist ja erkennbar, dass es in der Soziologie in Zeiten der Paradigmatase neue gesellschaftstheoretische Großentwürfe und eine hegemoniale Sozialtheorie schlicht nicht mehr gab, sodass man - aus Frankfurter Sicht - dem unübersichtlichen Theorieangebot einigermaßen orientierungslos gegenüberstand und gegenüberstehen musste. Aus Sicht der Soziologie bedeutete dies dann freilich auch, dass man je nach Theorieausrichtung die Schriften aus Frankfurt manchmal zur Kenntnis nahm, häufiger aber eben auch nicht. Die Versuche, aus einer normativistischen Position heraus die Gesellschaftsoder Sozialtheorie zu erneuern oder auch nur wiederzubeleben (einer der jüngeren Frankfurter Versuche war Axel Honneths Recht der Freiheit [2011]), scheiterten jedenfalls daran, dass entweder Ambitionen oder auch die Kraft zur Formulierung eigenständiger gesellschaftstheoretischer Entwürfe fehlten und die von den Frankfurtern immer wieder beschworenen Versuche einer Rekonstruktion der Geschichte,

\footnotetext{
3 Zur Debatte steht hier freilich nicht, ob man die von Mehring vorgeschlagenen Trennlinien genau so auch zeichnen muss.
} 
um dort für eine Gesellschaftstheorie relevante, aber verschüttete und für Kritik nutzbare Rationalitätspotenziale ausfindig zu machen, die es für die Gegenwart zu retten gelte, wenig plausibel waren: Mit Hegel, Durkheim und Parsons die Gegenwart des 21. Jahrhunderts aufschließen zu wollen, wie dies Honneth noch 2011 versucht hatte, konnte nur als höchst wagemutig bezeichnet werden, zumal die soziologischen Klassiker Durkheim und Parsons so sakrosankt nicht mehr sind und zudem auch die vom Ethnozentrismusverdacht motivierte Frage aufgeworfen wurde, wo genau nun Honneths Gegenwart geografisch anzusiedeln sei. Die Gesellschaftsoder Sozialtheorie lebt also ganz gut neben der normativistischen Politischen Theorie und Politischen Philosophie, ob sie von ihr lebt und von ihr viele positive und konstruktive Impulse zu erwarten hat, daran wird man zweifeln dürfen. Das gilt dann auch für die zumeist soziologisch inspirierten Zeitdiagnosen, deren Haltbarkeitsdatum ja auch immer kürzer wird und bei denen nicht zu erkennen ist, dass sie sich besonders intensiv der Politischen Theorie und Philosophie bedienen würden. Es ist im Übrigen auch eine falsche Vorstellung zu glauben, bei Zeitdiagnosen gehe es in erster Linie um empirische Richtigkeit und theoretische Stringenz und die Politische Theorie und Philosophie könnten oder sollten hier korrigierend tätig werden. Zeitdiagnosen sind selten empirisch unangreifbar und noch viel seltener theoretisch wirklich gut durchargumentiert. Vielmehr sind sie - so die plausible These von Fran Osrecki (2018, S. 471-475) - zu einer Art Theorieersatz geworden: Man einigt sich in verschiedenen Lagern der Soziologie darauf, dass irgendwo eine interessante Zeitdiagnose vorliegt und redet dann darüber. Zeitdiagnosen erfüllen eine Kommunikationsfunktion in einer zerklüfteten Wissenschaftslandschaft, weitergehende Ansprüche sollten und können sie auch oft nicht erheben. Kurz, vieles deutet darauf hin, dass der Normativismus Schwierigkeiten haben dürfte, Anschluss an soziologische oder sozialtheoretische Debatten zu finden.

Ob nun der politische Realismus aus der Soziologie heraus sehr viel mehr nachgefragt wird als der Normativismus? Dies dürfte schwer zu beantworten sein, tendenziell dann mit Ja, wenn es dieser Richtung der Politischen Theorie und Ideengeschichte darum geht, mehr sein zu wollen als Theoriegeschichte, sodass systematische Fragen und Wirkungszusammenhänge in den Mittelpunkt rücken. Dann wird Politische Theorie in der Tat interessant - zumindest für diejenigen in der Soziologie, die ohnehin eine gewisse Skepsis gegenüber allzu luftigen Gegenwartsdiagnosen und allzu wagemutig-abstrakten Gesellschaftstheorien hegen.

Heißt dies aber nun, dass Politische Theorie und Philosophie generell überhaupt nichts oder nur ganz wenig für die Soziologie bereitstellen könnten, dass Soziologinnen überhaupt nur wenig von diesen Disziplinen bzw. Subdisziplinen erwarten sollten? Nein, das heißt es nicht! Denn Politische Theorie und Philosophie, auch die Ideengeschichte, sind für die Soziologie dann besonders interessant, wenn - und hier ist das soeben angeführte Argument zuzuspitzen - die dort vorgelegten Analysen zentralen Annahmen der Soziologie widersprechen, diese relativieren, modifizieren etc. Die Soziologie selbst hat ja in ihrer Entwicklung als Disziplin eine ganze Menge an Theoremen und Fragen der neuzeitlichen Politischen Philosophie und Theorie mit aufgenommen - man denke an das Hobbessche Problem der Ordnung, an dem sich von Durkheim bis Parsons zahlreiche Soziologinnen abgearbeitet haben, man denke an die Problematiken der Arbeitsteilung und der sozialen Differenzierung, 
auf die besonders intensiv in der schottischen Moralphilosophie hingewiesen und die dann im 19. Jahrhundert von Karl Marx über Herbert Spencer bis hin zu Niklas Luhmann je unterschiedlich bearbeitet worden sind, oder man denke an Alexis de Tocquevilles Problemskizze zum Zusammenspiel von Freiheit, Individualismus und Gleichheit, wie dieses dann in den vorwiegend seit den 1980er-Jahren geführten sozialwissenschaftlichen Debatten um Gemeinwohlorientierung und Eigennutz zum Thema wurde. Die Soziologie hat sich also aus heutiger Sicht durchaus auch eine Menge an problematischem, jedenfalls nicht einfach zu schulterndem Ballast aufgeladen, womit ein jeder Versuch aus der Politischen Theorie, der Philosophie und der Ideengeschichte willkommen ist, diesen Ballast in selbstkritischer oder revisionistischer Weise zu prüfen und gegebenenfalls abzuwerfen.

Was das genau heißen kann, lässt sich an zwei Beispielen aus der anglo-amerikanischen Politischen Philosophie und Ideengeschichte kurz demonstrieren, die beide aus jeweils völlig unterschiedlicher Perspektive - implizit oder explizit - das extrem einflussreiche soziologische Theorem der sozialen oder funktionalen Differenzierung ins Visier genommen haben und damit unter Politischer Philosophie und Ideengeschichte mehr verstehen als nur die Hege und Pflege eines Archivs einmal entwickelter politischer Ordnungsvorstellungen. Moderne Gesellschaften sind ja - so lautet das Credo der klassischen Soziologie - funktional differenzierte Gesellschaften, und diese Einsicht fände sich schon bei den Klassikern, auch bei den Klassikern der schottischen Moralphilosophie, insbesondere bei Adam Smith. Für die Soziologie ist nun nicht interessant, wer sich alles im Umfeld von Smith, ob in Glasgow oder Edinburgh, getummelt hat, auf wen oder was Smith reagierte und wer wiederum ihn attackierte. Für eine an Grundsatzfragen interessierte Soziologie ist vielmehr interessant, ob die Deutung von Smith als einem der ersten Differenzierungstheoretiker so überhaupt stimmt, weil aus der diesbezüglichen Einordnung eine ganze Menge an Konsequenzen zu ziehen sind. Was das bedeutet, hat vor fast 15 Jahren der mittlerweile verstorbene ungarisch-britische Politikwissenschaftler István Hont (2005, S. 8) mit Blick auf Smiths Wohlstand der Nationen demonstriert, indem er darauf aufmerksam machte, wie eng Smith in jenem Zentralwerk der Politischen Ökonomie und frühen Sozialwissenschaften an David Humes Überlegungen zur militärischen Überlebensfähigkeit von Staaten anknüpfte. Überzeugend legte Hont dar, dass Smiths Buch ganz überwiegend keines ,,about perpetual peace“, sondern eines über „,competitive economic strategy“ gewesen sei. Es sei kein Zufall - so Hont -, dass das zentrale Kapitel I des fünften Buches („,Die Finanzen des Landesherrn oder des Staates“) sofort mit Reflexionen über „Ausgaben für die Landesverteidigung“ beginnt und mit Überlegungen zur gleichzeitigen oder parallelen Entwicklung von gesellschaftlichen und militärischen Strukturen anschließt. Hont zufolge ist Smiths Argumentation im Wohlstand der Nationen somit kein Versuch einer lediglich deskriptiven Fassung der zentralen Strukturen einer (,modernen“) Marktgesellschaft, keine bloß theoretische Reflexion über ökonomische Effizienz, sondern ein zutiefst politisches Unterfangen - geprägt vom militärisch-imperialen Kontext ,seines“ Landes und seiner Zeit. Wenn man dies zuspitzen will, so war Smiths Wohlstand der Nationen ein Plädoyer zur Forcierung einer ,ganz spezifischen“ und damit historisch kontingenten Form der Differenzierung zwischen Staat und Markt, um die internationale und insbesondere militärische Wettbewerbsfähigkeit des Staates, hier 
des britischen, zu erhöhen. ${ }^{4}$ Hier wird also Politische Theorie und Ideengeschichte in systematischer Absicht betrieben mit entsprechend hoher Rezeptionsbereitschaft unter Soziologinnen.

Ein sehr viel jüngeres Beispiel ist das Buch von Quinn Slobodian mit dem Titel Globalists (2018). Für Soziologinnen ist dieses ideengeschichtliche Buch schlicht deswegen anregend und eine Provokation, weil es mit den Vorstellungen bricht, dass (a) die ordnungspolitischen und ökonomischen Entwurfe der frühen Neoliberalen wie Hayek oder Röpke irgendetwas mit Individualismus zu tun gehabt hätten, (b) dass es diesen Theoretikern in irgendeiner Weise um Demokratie gegangen wäre, und (c) dass das neoliberale Weltbild überhaupt in erster Linie ein ökonomisches gewesen sei. Slobodian zeigt vielmehr, dass das Denken von Hayek und seinen Mitstreitern Ausdruck einer von einer spezifischen historischen Konfliktsituation der Zwischenkriegszeit geprägten Rechts- und Staatsvorstellung war, die man verstehen muss, um der Dynamik der neoliberalen Attacken der 1970er-Jahre gerecht werden zu können. Anders formuliert: Nicht wenige der vermeintlichen Helden der ökonomischen Theorie der Zwischenkriegszeit werden in der Perspektive Slobodians eigentümliche Figuren mit einer spezifischen politischen Agenda, was der Soziologie wiederum hilft, Differenzierungsfragen neu zu stellen und einzuordnen, mithin die eigene Fachgeschichte mit den immer weiter tradierten Theoremen (hier demjenigen der Differenzierung von Staat und Markt) kritisch zu reflektieren.

Zusammenfassend lässt sich vielleicht mein Argument so formulieren: Politische Theorie und Philosophie bzw. die Politische Ideengeschichte werden für die Soziologie nicht dann interessant, wenn sie auf das große Ganze (die Gesellschaftstheorie) zielen, sondern wenn sie es mit bestimmten Middle-Range-Theoremen aufnehmen, seien dies nun „Differenzierung“ oder eben andere Prozessbegriffe wie „Säkularisierung“, „Bürokratisierung“, „Individualisierung“ etc., von denen man sich aus soziologischer Sicht wünschen würde, sie würden in der Politischen Ideen- und Theoriegeschichte sehr viel stärker - systematischer und kritischer - in den Mittelpunkt rücken, um der Soziologie auf die Sprünge zu helfen.

\section{Replik: Selbstvergewisserung hilft nicht}

\section{Claudia Landwehr}

Die ,eigenartige Mischung aus gefühlter Überlegenheit und gefühlter mangelnder Wertschätzung“ in der Politischen Theorie und Ideengeschichte, die Michael Zürn in seinem Beitrag diagnostiziert, ist die klassische Symptomatik narzisstischer Kränkung. Diese Gefühlslage scheint besonders in den Geisteswissenschaften weit verbreitet. Ein Doktorand der Anglistik an der Harvard University hat kürzlich in einem gleichermaßen witzigen wie scharfsinnigen Essay beschrieben, wie diese Gefühlslage eine „Reassurance Lit“" hervorbringe, mit der sich Geisteswissenschaftler

\footnotetext{
${ }^{4}$ Darauf verweist auch die Tatsache, dass gerade auch angesichts aktueller politischer und gesellschaftlicher Entwicklungen die Differenzierungstheorie längst nicht mehr so unumstritten ist, wie dies noch bis in die 1980er-Jahre hinein der Fall war.
} 
beständig ihrer eigenen Relevanz vergewissern: „We remind ourselves repeatedly that what we do is difficult, heroic, and critically undervalued" (Tyson 2020). Dass solche Selbstvergewisserung allenfalls ein schwacher Trost bleibt, zeigt sich schon daran, dass sie ständig wiederholt werden muss. Zugleich wird sie wenig dazu beitragen, vorrangig geisteswissenschaftlich arbeitende Forscherinnen - und in diesem Fall konkret die Politische Theorie - im Wettbewerb mit anderen Fächern und Teildisziplinen zu stärken.

Dass die Theorie-Sektion zu dieser Diskussion kritische Außenperspektiven aus der Vergleichenden Politikwissenschaft, den Internationalen Beziehungen und der Soziologie erbeten hat, zeigt, dass man sich dieses Problems bewusst ist. Auch ich habe kein Interesse an weiterer Selbstvergewisserung und schließe mich der Kritik der Fachkollegen am Zustand der Politischen Theorie weitestgehend an. Gleichzeitig sollten wir aber über die kritische Bestandsaufnahme hinausgehen und konstruktiv nach Strategien und Maßnahmen fragen, die uns helfen können, die Politische Theorie zu stärken. Hierbei darf es nicht nur darum gehen, die Berufungsaussichten für jüngere Kolleginnen zu verbessern. Für Theoretikerinnen ist dies zweifelsohne ein professionspolitisch wichtiges Motiv, für Vertreterinnen anderer Disziplinen und Teilbereiche aber kein Grund, Theorie-Professuren zu erhalten und auszubauen. Vielmehr müssen wir sie davon überzeugen, dass es darum geht, mit einer starken Politischen Theorie und Ideengeschichte die angemessene Ausbildung unserer Studierenden, unter denen viele zukünftige Lehrerinnen und damit gesellschaftliche Multiplikatorinnen sind, sowie den Anspruch und die Qualität politikwissenschaftlicher Forschung sicherzustellen.

Wie Armin Schäfer feststellt, fehlt es vielen Theoretikerinnen an einem Grundverständnis für empirische Methoden, welches Voraussetzung für die von Michael Zürn angemahnte Anschlussfähigkeit zwischen normativen, pragmatischen und empirischen Zugängen wäre. Dieses fehlende Methodenverständnis wird zum Problem, wenn Theoretikerinnen zugleich darum bemüht sind, ihre Argumente auf empirische Befunde zu stützen, dabei aber nicht selten selektiv und unkritisch vorgehen. Es hat daher seinen guten Sinn, dass immer mehr politikwissenschaftliche Institute, die ein grundständiges politikwissenschaftliches Studium anbieten, heute eine eigene Methodenprofessur haben. Wovon wir Kolleginnen aus anderen Teilbereichen und Hochschulleitungen überzeugen müssen, ist, dass eine Theorieprofessur genauso unverzichtbar ist. Wo die Ausbildung in Politischer Theorie und Ideengeschichte leidet, geht begriffliche Präzision verloren, verschwimmen normative Bewertungsmaßstäbe, und es fehlt oft auch die Fähigkeit zur Einordnung empirischer Fragestellungen und Befunde hinsichtlich ihrer praktisch-politischen und normativen Relevanz. Entsprechende Fähigkeiten sollten nicht nur Studierenden vermittelt werden: auch empirisch arbeitende Kolleginnen können in dieser Hinsicht von einem stärkeren Austausch mit Theoretikerinnen und einer höheren Wertschätzung von Theoriearbeit profitieren. Denn genauso wenig, wie empirische Befunde unkritisch oder selektiv rezipiert werden dürfen, können normative Begriffe und Bewertungsmaßstäbe theoretisch unreflektiert und ,aus dem Bauch heraus“ verwendet werden - und genau dies geschieht, wenn die Politische Theorie und Ideengeschichte in Lehre und Forschung vernachlässigt wird. 
Richtig scheint mir aber auch die Beobachtung, dass viele deutsche Politiktheoretikerinnen vergleichsweise selten auf internationalen Konferenzen anzutreffen sind und überwiegend für ein deutschsprachiges Publikum schreiben. Dies steht in einem interessanten Widerspruch zu der Tatsache, dass Theoretikerinnen aus dem englischen (und teilweise auch französischen) Sprachraum in der deutschen Politischen Theorie breit und intensiv rezipiert werden. Wenn viele deutsche Autorinnen trotzdem vergleichsweise wenig international publizieren, liegt das sicher nicht daran, dass es keine passenden Publikationsorte gäbe. Zeitschriften wie das American Political Science Review, die sich an das gesamte Fach richten, publizieren regelmäßig Aufsätze aus der Politischen Theorie. Zudem gibt eine Reihe sehr sichtbarer TheorieZeitschriften (Political Theory, The Journal of Political Philosophy, Constellations u. a.), die zudem dank professionalisierter Redaktion, und vermutlich auch entsprechenden Algorithmen, meist passende und kompetente Gutachterinnen finden. Mein Eindruck ist, dass nicht wenige Kolleginnen vielmehr unwillig sind, Kritik von Gutachterinnen anzunehmen und sich der mühsamen Aufgabe zu stellen, eigene Texte immer wieder zu überarbeiten, um Argumente zu schärfen und richtig verstanden zu werden. Wer in internationalen und begutachteten Fachzeitschriften publizieren will, kann deshalb insgesamt weniger publizieren als diejenigen, die bevorzugt in Sammelbänden veröffentlichen. Bei der Publikationsstrategie auf „,messbare“ Qualität statt auf Quantität zu setzen, könnte aber den Kolleginnen aus anderen Teilbereichen zeigen, dass wir uns an denselben Maßstäben messen lassen wie sie und bereit sind, uns dem Urteil der Peers zu stellen. Dass internationales Publizieren in der Politischen Theorie möglich ist, zeigt auch der Erfolg von Kolleginnen aus der praktischen Philosophie, die hier deutlich weiter sind und für politikwissenschaftliche Theoretikerinnen zunehmend zur Konkurrenz in Berufungsverfahren werden (was umgekehrt definitiv nicht der Fall ist).

Zuletzt möchte ich aber noch einige skeptische Überlegungen zur öffentlichen Sichtbarkeit und interdisziplinären Nützlichkeit der Politischen Theorie formulieren. Armin Schäfer hat recht, wenn er feststellt, dass etwa die Wahl- und Einstellungsforschung von den Medien sehr viel stärker nachgefragt wird als die Theorie: Gerade in unsicheren Zeiten wächst die Sehnsucht nach harten und vermeintlich objektiven Zahlen. Zugleich sehe ich durchaus immer wieder Kolleginnen, die unsere Teildisziplin erfolgreich in der Öffentlichkeit vertreten. Allerdings geht es dann seltener darum, eigene Forschungsergebnisse zu präsentieren, als darum Angebote zur Einordnung und Bewertung aktueller Entwicklungen und Ereignisse zu machen. Was die eigene Forschung angeht, wissen Politiktheoretikerinnen natürlich, dass es in der Wissenschaft und Wissenschaftskommunikation keinesfalls so ist, dass sich jedes Angebot seine öffentliche und mediale Nachfrage selbst schafft. Aber das ist auch gut so: Politische Theorie ist kein Konsumgut. Gerade die so wichtige Kritik rechtfertigt sich nicht aus der Nachfrage, sondern aus den Missständen und Problemen, die sie adressiert. Deshalb sollten wir uns nicht dazu hinreißen lassen, mit Blick auf öffentliche Sichtbarkeit ,nachfrageorientiert“ zu forschen.

Dasselbe gilt für die auch in der Forschungsförderung immer wichtiger werdende interdisziplinäre Kooperation. Die Politikwissenschaft insgesamt und die Politische Theorie im Besonderen dürfen sich hier nicht zur Hilfswissenschaft degradieren lassen und sich als geisteswissenschaftliches Feigenblatt für lebens- oder naturwis- 
senschaftliche Großprojekte anbieten. Interdisziplinäre Zusammenarbeit muss auf Augenhöhe erfolgen. Synergieeffekte entstehen dort, wo Methoden und Zugänge komplementär sind und unterschiedliche disziplinäre Perspektiven auf dieselben Phänomene eine vollständigere Erfassung und Bewertung dieser ermöglichen. In den vergangenen Jahren hat das Erstarken des Populismus zu einem Boom der entsprechenden Forschung geführt, der empirische Politikwissenschaft und Politische Theorie verstärkt miteinander ins Gespräch gebracht hat. In den kommenden Jahren könnten und sollten die empirische und die theoretische Politikwissenschaft gemeinsam einen Beitrag dazu leisten, Regierungsentscheidungen in der CoronaKrise einzuordnen und $\mathrm{zu}$ bewerten und gemeinsamen mit anderen Fächern die wirtschaftlichen, sozialen und politischen Folgen der Krise kritisch zu analysieren.

\section{Replik: Selbsterkenntnis des Wissenschaftssystems}

\section{Regina Kreide}

Die Selbstverständigung über die eigene Disziplin erfasst in regelmäßigen Abständen den wissenschaftlichen Alltag. Sie zeugt nicht notwendigerweise von einer Krisenstimmung in der jeweiligen Disziplin (Was wurde versäumt?), sondern kann auch auf Aufbruch und Veränderung hinweisen (Was muss sich ändern?). Die allesamt erhellenden Beiträge von Wolfgang Knöbl, Armin Schäfer und Michael Zürn konfrontieren die Politische Theorie und Ideengeschichte - zugespitzt gesagt - mit der Einsicht, dass sie zu häufig in den Spiegel blickt und das auch nur, um sich von der eigenen Vollkommenheit zu überzeugen. Selbstkritik und eine ernsthafte Auseinandersetzung mit historischen Versäumnissen und Veränderungspotenzialen sieht anders aus, so der Tenor. Ein zurecht erhobener Vorwurf! Allerdings scheinen mir Versäumnisse nicht nur bei jenen zu liegen, die sich der Politischen Theorie zugehörig fühlen. Auch die Kritikpunkte, so plausibel sie im Einzelnen sein mögen, greifen letztlich zu kurz. Aus allen drei Beiträgen spricht eine spezifische Auffassung dessen, was eine wissenschaftliche Disziplin auszeichnet. Unterstellt wird, dass es sinnvoll ist, trennscharfe disziplinäre Zuordnungen $\mathrm{zu}$ betreiben und eine klare Bestimmung vorzunehmen, was - in diesem Fall - zur Politischen Theorie gehört und was eben nicht. Daraus spricht - man möge mir die Polemik verzeihen - eine gewisse „Disziplinversessenheit“, die einer kritischen Analyse und Beurteilung komplexer gesellschaftlicher Verhältnisse im Wege stehen kann.

Interessanterweise fordern alle drei Autoren, dass sich die Politische Theorie in der einen oder anderen Weise der empirischen Forschung öffnen und disziplinäre Grenzen überschreiten sollte. Auch diese Meinung teile ich. Aber jene immer noch recht schmal angelegte Transzendierung wissenschaftlicher Grenzen - von der normativen Reflexion hin zu einer stärkeren empirischen Ausrichtung - ist keinesfalls ausreichend, um gegenwärtige Probleme angemessen analysieren zu können. Zudem ist damit auch noch nichts darüber gesagt, wie das Zusammenspiel von Empirie und Normativität aussehen könnte. In der gebotenen Kürze möchte ich darlegen, dass es angesichts globaler gesellschaftlicher Verflechtungen und Bedrohungen hilfreich, wenn nicht sogar geboten wäre, disziplinär definierte Bereiche nur als institutionelle 
Ausgangsplattformen zu sehen, die wissenschaftliche Arbeit jedoch gemeinsamer Analyse gesamtgesellschaftlicher Tendenzen zu widmen. Das wäre eine Weichenstellung, die den Blick auf Forschungsfragen von vorneherein verändert. Zwei Annahmen, die zum einen die disziplinäre Grenzziehung und zum anderen das TheoriePraxis-Verhältnis betreffen, liegen meinen Überlegungen zugrunde.

Zum einen spiegelt die Einteilung des Wissenschaftsbetriebs nicht die notwendigen wissenschaftlichen Problemanalysen und Herangehensweisen wider. Tatsächlich sind wissenschaftliche Disziplinen - wie etwa schon Bruno Latour und Steve Woolgar (1986) gezeigt haben - künstlich geschaffene Formen der Wissensorganisation, das heißt, sie sind das Ergebnis von Machtverhältnissen und Karrierestrategien und meistens weit davon entfernt, sich über eine gesamtgesellschaftliche Problembearbeitung zu definieren. Die in rascher Folge auftretenden jüngsten Krisen zeigen, dass weder Finanz- und Wirtschaftsexpertinnen für die Finanzkrise noch Virologinnen (wobei es in den allermeisten Fällen Männer sind) und andere medizinische Expertinnen für die „Corona-Krise“, so wichtig wie ihre Meinung auch in der jeweiligen Krise sein mag, alleine in der Lage sind, die Situation angemessen analysieren und bewerten können - was die meisten von ihnen auch ganz offen zugeben. Gesellschaftswissenschaftliche, psychologische, ethische, künstlerische Perspektiven wären wünschenswert, um ein breiteres Bild über Ursachen der Krisen und Folgen für die verschiedenen Bevölkerungsgruppen sowie, ganz wichtig, die möglichen Maßnahmen zu deren Überwindung zu erhalten.

Für einen der komplexen Situationen angemessenen Erkenntnisgewinn ist es unerlässlich, dass sich wissenschaftliche Disziplinen füreinander und für die Einsichten und Methoden der anderen öffnen. Im Idealfall ergibt sich daraus ein interdisziplinäres Zusammenarbeiten, in dem man sich ausgehend von gesellschaftlichen Problemen um Analysen, Kritik und emanzipative Lösungen bemüht. Das erfordert von Anfang an, am besten bereits mit Beginn des Studiums, dass umfangreiches Wissen über gesamtgesellschaftliche Prozesse und eine Pluralität von Methoden gelehrt wird, um eine habituelle Offenheit für andere Disziplinen zu gewinnen. Dann werden sich letztlich die verschiedenen Wissenselemente und wissenschaftlichen Praktiken als Teile einer Gesamttheorie begreifen können.

Zweitens greift selbst eine bloße engere Verzahnung normativer und empirischer Forschung immer noch zu kurz, denn das allein reflektiert nicht in ausreichendem Maße das erforderliche Verhältnis zwischen Theorie und Praxis. Theoriebildung ohne den Bezug zur Praxis ist aber schlechterdings nicht denkbar bzw. schlechte Theorie. Das ist auch unter den drei Autoren unstrittig. Wie aber sollte der ,Bezug zur Praxis' aussehen? Theorie selbst muss sich, wie Max Horkheimer hervorhebt, als einen Teil des Lebenszusammenhangs begreifen, den sie zu erfassen versucht (etwa Horkheimer 1937). Damit reflektiert Theorie von vorneherein ihre eigenen Bedingungen und versteht sich als Teil der Praxis, die sie analysiert. Das hat nicht nur Folgen für die Theorie, sondern eben auch für die Praxis. Konsequenterweise nämlich können Phänomene wie Ausbeutung, Naturzerstörung, Entfremdung, Ausgrenzung nicht in der Theorie, sondern nur in der Praxis überwunden werden. Wissenschaft wird in diesem Verständnis selbst zu einer Praxis, die nicht nur beschreibt, erklärt und begründet, sondern Lösungen vorschlägt, die die Verhältnisse verändern könnten. 
Auch historisch betrachtet ist diese Auffassung des praktischen Bezugs von Wissenschaft durchaus plausibel. Es gibt keine für alle Zeiten festgelegte stabile Ordnung des Wissens oder einen privilegierten Zugang zum Wissen, durch den wissenschaftliche Maßstäbe und Disziplinen für immer festlegt wären. Die Organisation wissenschaftlicher Erkenntnisse ist historisch gewachsen und stets in den sozialen und politischen Kontext eingebunden. In bestimmten Phasen erlangten bestimmte gesellschaftliche Probleme und ihre wissenschaftliche Bearbeitung besondere Bedeutung: die Religionskritik war es während des 18. Jahrhunderts, etwas später die Kritik der politischen Ökonomie, die Auseinandersetzung mit Technik und Naturwissenschaften stach im 20. Jahrhundert hervor, ebenso wie gegenwärtig die Kritik an weltweiter Ungleichheit, am Klimawandel, Antiliberalismus und Rassismus. Die Vielschichtigkeit und Verwobenheit globaler Probleme erfordert eine immer wieder aufs Neue auszutarierende Beziehung zwischen Theorie und Praxis, die über eine bloße Öffnung der Politischen Theorie hin zur Empirie und die Integration empirischer Arbeiten in die normative Theoriebildung hinausreicht. Die Selbsterkenntnis von Wissenschaft kann also insofern als emanzipatorisch bezeichnet werden, als sie uns von fremdbestimmter Herrschaft und illegitimen Machtverhältnissen befreien kann. Erkenntnisgewinn erfolgt durch gesellschaftliche Praxis.

Letztlich, so lässt sich sagen, erfordert die Selbstverständigung über das eigene wissenschaftliche Tun einer Disziplin, wie es die Politische Theorie ist, eine Reflexion darüber, wie die Einteilung in wissenschaftliche Disziplinen zustande gekommen und wie eine praxisbasierte Bearbeitung globaler Probleme möglich ist. Die „Disziplinierung“ des Wissenschaftsbetriebes kann dem wissenschaftlichen Erkenntnisgewinn auch im Wege stehen. Diskurse über die wissenschaftliche Identität der eigenen Disziplin lassen sich auch als notwendiger Bestandteil eines jeden wissenschaftlichen Arbeitens begreifen. Was nämlich wäre Wissenschaft ohne die regelmäßige kritische Hinterfragung des eigenen Tuns und Urteilens - was bislang nur einige Stränge der Politischen Theorie und Ideengeschichte betreiben? Eine kritische Reflexion der eigenen wissenschaftlichen Maßstäbe, der inhaltlichen Fokussierung, der verwendeten Methoden und vor allem der emanzipativen Stoßrichtung ist für jeden wissenschaftlichen Diskurs unumgänglich. Die hier geforderte Interdisziplinarität ist nicht interdisziplinär in dem Sinne, dass jede beteiligte Wissenschaft aus ihrer professionellen Sicht auf einen geteilten Gegenstand blickt und diesen analysiert. Gefragt ist gegenwärtig vielmehr ein Wissenschaftsverständnis, das sich der eigenen Spezialisierung, der eigenen Stärken und Kenntnisse bewusst ist, aber gerade aus dem Wissen um diese Beschränkung für das Wissen und methodische Vorgehen der anderen Disziplinen offen ist und so ein gemeinsam identifiziertes Problem im gesamtgesellschaftlichen Kontext gemeinsam bearbeitet werden kann. Ich bin nicht einmal sicher, dass die drei Autoren mir widersprechen würden, denn alle drei stehen, auch gerade durch ihre institutionelle Einbindung in interdisziplinär arbeitende Forschungsinstitute, für ein transdisziplinäres Wissenschaftsverständnis. Womöglich sind auch sie der Ansicht, dass nicht allein mehr empirische Forschung, sondern mehr wissenschaftliche Grenzüberschreitungen gegenwärtig gefragt sind. Der Politischen Theorie würde diese Offenheit in jedem Fall guttun. 


\section{Replik: Methodenpluralismus und Öffentlichkeit}

\section{Eva Marlene Hausteiner}

Als besonders heterogene Disziplin tritt die Politikwissenschaft zwar nach außen als ein Fach auf, nach innen aber hat sie es mit klassisch interdisziplinären Problemen zu tun: Wie können wir trotz unterschiedlicher Methoden produktiv kommunizieren, wie verbinden wir unsere politikwissenschaftliche Analyse mit begriffskritischer Reflexion, und wie gelingt Zusammenarbeit unter Beibehaltung unserer jeweiligen disziplinären Spezifik? Diese innerfachliche Diversität bringt freilich auch ein großes Privileg mit sich, nämlich einen anhaltenden Dialog mit Forscherinnen, mit denen uns besonders viel verbindet - nicht nur ein enges institutionelles Band, sondern auch das gemeinsame Interesse, Politik und das Politische zu verstehen.

Als Politiktheoretikerinnen sollten wir also fachfamiliäre Hinweise besonders ernst nehmen. Armin Schäfer, Michael Zürn und Wolfgang Knöbl formulieren berechtigte Anregungen, die in unserer Teildisziplin auf offene Ohren treffen werden. Im Sinne eines anhaltenden Dialoges greife ich aber im Folgenden zwei Punkte heraus: Die asymmetrische gegenseitige Wahrnehmung methodischer Praxen sowie das angebliche Manko öffentlicher Debattierfreude. In beiden Fällen will ich argumentieren, dass Politiktheoretikerinnen aller Façon den genannten Herausforderungen längst engagiert begegnen - aber auch, dass wir aus guten Gründen auf bestimmten Spezifika der Politischen Theorie und Ideengeschichte beharren.

Jede Politiktheoretikerin ist gut vertraut mit der Außenwahrnehmung, unsere Teildisziplin pflege ein eher separiertes, möglicherweise auch weniger professionalisiertes Methodenverständnis. Schon hieraus, so argumentiert Schäfer, ergebe sich eine unglückliche kommunikative Kluft: Wie kann, so die wichtige Frage, der Kontakt der Politischen Theorie und Ideengeschichte zu den eher quantitativ-empirisch arbeitenden Zweigen des Fachs gelingen?

Die enorme methodische Diversität in den Sozialwissenschaften gehört zu ihren großen Stärken, führt aber zweifellos oft zu nur mühselig überwindbaren Übersetzungsbarrieren. Das hat auch mit der asymmetrischen Wahrnehmung der in den Teildisziplinen üblicherweise praktizierten Methoden zu tun: Die hermeneutischen Werkzeuge, die in der Politischen Theorie und Ideengeschichte zur Anwendung kommen, sind bisweilen ,,von außen“ für Fachfremde besser verständlich als umgekehrt jene quantitativ-empirischen Instrumente, die in anderen Teildisziplinen gängiger sind.

Es wäre jedoch ein Missverständnis, daraus abzulesen, die politiktheoretische Methodologie sei darum weniger avanciert, weil sie weniger hermetisch daherkommt. Entgegen der von Michael Zürn beobachteten ,methodischen Homogenität“ zeichnet sich unsere Teildisziplin durch einen bemerkenswerten Methodenpluralismus aus, der immer wieder Gegenstand einer durchaus kontroversen Verständigung ist. ${ }^{5}$ Zudem nimmt seit geraumer Zeit auch bei uns eine anspruchsvolle Methoden(aus)bildung einen hohen Stellenwert ein. Die Politische Ideengeschichte stellt

\footnotetext{
5 Zum politiktheoretisch-ideengeschichtlichen Methodenpluralismus siehe Busen und Weiss (2013) sowie Dryzek et al. (2008).
} 
hierfür ein gutes Beispiel dar: Aus den großen Schulen der Ideengeschichte wie der Cambridge School, der Begriffsgeschichte oder der Genealogie nach Foucault, die sich in den vergangenen zwei Jahrzehnten deutlich von ihren nationalen Herkunftskontexten emanzipiert haben, haben sich neue Zugriffe herausgebildet: ideologiekritische Genealogie, begriffsfokussierte Diskursanalyse, realistische Challenge-undResponse-Analyse oder auch die interkulturell vergleichende politische Theorie. ${ }^{6}$ Diese Debatten sind in hohem Maße dynamisch und internationalisiert - auch wenn gerade in der Politischen Ideengeschichte weiterhin viel auf Deutsch publiziert wird. Dass neben Englisch nach wie vor in deutscher Sprache (oder auch in anderen Sprachen) publiziert wird, hat übrigens nicht allein mit dem Anspruch auf präzise argumentative Darstellung zu tun. Es geht vielmehr einerseits darum, originalsprachlichen Quellen gerecht zu werden. Andererseits sollen plurale Adressatenkreise angesprochen werden. Von deutschsprachiger Publikationstätigkeit profitiert dabei auch die von Schäfer angemahnte Adressierung breiterer nichtakademischer Öffentlichkeiten.

Dank der praktizierten Gleichzeitigkeit von Methodenpluralismus und methodischer Spezialisierung kennt die Politische Theorie und Ideengeschichte die Herausforderung interdisziplinärer Kommunikation also bereits aus dem eigenen Arbeitsgebiet. Der rege Austausch innerhalb unserer DVPW-Sektion dient nicht zuletzt dieser laufenden „trans-sub-disziplinären“ Selbstverständigung. Daraus ergibt sich ein besonderer Vorzug: Dass politiktheoretische Forschung - trotz ihrer anspruchsvollen Methodik - oft für Fachfremde recht gut verständlich ist, hat nicht nur mit ihrem hermeneutischen, sprach- statt datenbasierten Charakter zu tun, sondern auch mit der Einübung darin, den eigenen methodischen Zugriff gegenüber alternativen Ansätzen zu begründen und den anderen Forscherinnen auf nachvollziehbare Weise zu kommunizieren. Die Strategie kann daher nur sein: Eine Internationalisierung ist notwendig, aber nicht auf Kosten der eigenen methodischen Spezifik und Fachkultur. Der Appell, sich dabei weiterhin um eine verständliche Kommunikation nach außen zu bemühen, gilt freilich auch für unsere Kolleginnen aus den eher quantitativ arbeitenden Bereichen: also ihre Ansätze für die sozialwissenschaftliche Nachbarschaft und darüber hinaus zugänglich zu kommunizieren und vice versa den hohen Anspruch des qualitativen Methodenspektrums anzuerkennen und sich mit diesem auseinanderzusetzen.

Das monierte Defizit in der Öffentlichkeitswirksamkeit der Politischen Theorie und Ideengeschichte steht ganz offensichtlich im Widerspruch zu unserer Selbstwahrnehmung als kommunikativ erfahrene Wissenschaftlerinnen: Wenn es nämlich zutrifft, dass wir in der Kommunikation unserer Erkenntnisinteressen und Antwortversuche versiert sind, weshalb ist unser Teilbereich dann - wie Armin Schäfer argumentiert - in der öffentlichen Debatte nur spärlich vertreten? Aber trifft diese Kritik zu? Mir erscheint die empirische Grundlage dieser Einschätzung unklar, denn die Debattenfreudigkeit gerade vieler noch nicht berufener Wissenschaftlerinnen fällt auf. Dies ist vor allem deshalb bemerkenswert, weil in der deutschen Wissenschaft breitenöffentliches Engagement weiterhin nicht immer als karriereförderlich wahr-

\footnotetext{
${ }^{6}$ Die Bandbreite aktueller ideengeschichtlicher Zugänge wird umfassender dargestellt in Salzborn (2018), Beckstein und Weber (2014) sowie Bluhm und Gebhardt (2006).
} 
genommen wird. Zu den drängendsten Debatten unserer politischen Gegenwart, von der Migrationspolitik über die Klimakatastrophe bis hin zu geschlechterpolitischen Debatten nehmen gerade jüngere Vertreterinnen unserer Teildisziplin immer wieder öffentlichkeitswirksam Stellung - in klassischen Printmedien und den ÖffentlichRechtlichen, aber auch in der digitalen Sphäre. Begriffliche Schärfung, historische Perspektivierung und normativ fundierte Kritik - allesamt Stärken der Politischen Theorie und Ideengeschichte - sind offenbar gefragt. An der seit März die Aufmerksamkeit monopolisierenden Pandemiesituation ist dies durchaus abzulesen: Für die historisierende Dekonstruktion der Rhetoriken von Krieg und Ausnahmezustand, die Suche nach den Wurzeln der nun sichtbaren Folgen des Neoliberalismus und die kritische Prüfung der ubiquitären Verweise der Politik auf die ,unpolitische“ Expertise der Wissenschaft ist unsere Teildisziplin prädestiniert und nachgefragt. Nicht zuletzt gehören das Wirken von Intellektuellen und die kritische Reflexion politikwissenschaftlicher Politikberatung zu den „Stammthemen“ der Politischen Theorie und Ideengeschichte. Zurecht betont Wolfgang Knöbl die Fähigkeit der Politischen Theorie und insbesondere der Ideengeschichte, etablierte Annahmen und dominante historische Narrative kritisch zu prüfen - das damit einhergehende Provokationspotenzial hat sich als überaus debattentauglich erwiesen.

Soll es freilich künftig noch mehr politiktheoretische und ideengeschichtliche Wortmeldungen gerade jüngerer Wissenschaftlerinnen $\mathrm{zu}$ gesellschaftspolitischen Themen geben, dann liegt ein aussichtsreicher Weg in der Förderung nichtprekärer Beschäftigungsverhältnisse. Eine Verbesserung struktureller Bedingungen wäre sicherlich hilfreich, damit Politiktheoretikerinnen ihre begriffliche, historische und kritische Perspektive noch mehr in die öffentliche Debatte einbringen. Momentan erleben wir allerdings eher das Gegenteil: nämlich einen sukzessiven Rückbau der Politischen Theorie und Ideengeschichte an vielen Instituten - und das, obwohl die Bedeutung unserer Teildisziplin für die Politikwissenschaft als Demokratiewissenschaft (wie auch vielerorts ganz konkret etwa für eine fundierte Lehramtsausbildung) kaum infrage steht. Soll die Politische Theorie und Ideengeschichte weiter wirksam ihren Beitrag zur demokratisch-gesellschaftlichen Selbstreflexion leisten, kommt man an der Ressourcenfrage nicht vorbei. Statt einer machtpolitisch verkürzten, die mittel- und langfristigen Folgen aus dem Blick verlierenden Ressourcenkonkurrenz sollten wir daher - so mein Vorschlag - die produktiven innerfachlichen Auseinandersetzungen und Verständigungsprozesse dafür nutzen, das Fach in seiner Heterogenität zu stärken und zu integrieren. Dazu gehört auch, gemeinsam dafür zu sorgen, dass in der Arbeitsteilung der Sozialwissenschaften die grundlegende, kritisch-reflexive sowie historisch perspektivierende Rolle der Politischen Theorie und Ideengeschichte weiterhin fest verankert ist.

Funding Open Access funding enabled and organized by Projekt DEAL.

Open Access Dieser Artikel wird unter der Creative Commons Namensnennung 4.0 International Lizenz veröffentlicht, welche die Nutzung, Vervielfältigung, Bearbeitung, Verbreitung und Wiedergabe in jeglichem Medium und Format erlaubt, sofern Sie den/die ursprünglichen Autor(en) und die Quelle ordnungsgemäß nennen, einen Link zur Creative Commons Lizenz beifügen und angeben, ob Änderungen vorgenommen wurden. 
Die in diesem Artikel enthaltenen Bilder und sonstiges Drittmaterial unterliegen ebenfalls der genannten Creative Commons Lizenz, sofern sich aus der Abbildungslegende nichts anderes ergibt. Sofern das betreffende Material nicht unter der genannten Creative Commons Lizenz steht und die betreffende Handlung nicht nach gesetzlichen Vorschriften erlaubt ist, ist für die oben aufgeführten Weiterverwendungen des Materials die Einwilligung des jeweiligen Rechteinhabers einzuholen.

Weitere Details zur Lizenz entnehmen Sie bitte der Lizenzinformation auf http://creativecommons.org/ licenses/by/4.0/deed.de.

\section{Literatur}

Allen, Amy, und Eduardo Mendieta (Hrsg.). 2019. The Cambridge Habermas Lexicon. Cambridge: Cambridge University Press.

Beckstein, Martin, und Ralph Weber (Hrsg.). 2014. Politische Ideengeschichte. Interpretationsansätze in der Praxis. Göttingen: UTB.

Bleek, Wilhelm. 2001. Geschichte der Politikwissenschaft in Deutschland. München: C.H. Beck.

Bluhm, Harald, und Jürgen Gebhardt (Hrsg.). 2006. Politische Ideengeschichte im 20. Jahrhundert. Konzepte und Kritik. Baden-Baden: Nomos.

Busen, Andreas, und Alexander Weiss (Hrsg.). 2013. Ansätze und Methoden zur Erforschung politischen Denkens. Schriftenreihe der Sektion Politische Theorie und Ideengeschichte der Deutschen Vereinigung für Politikwissenschaft. Baden-Baden: Nomos.

Dahl, Robert A. 1989. Democracy and its critics. New Haven: Yale University Press.

Dryzek, John S., Bonnie Honig, und Anne Phillips (Hrsg.). 2008. The oxford handbook of political theory. Oxford: Oxford University Press.

Ellersiek, Helke. 2019. Politische Vermessung der Welt. tageszeitung. https://taz.de/Theorie-versusEmpirie/!5635627. Zugegriffen: 10. Sept. 2020.

Forrester, Katrina. 2019. In the shadow of justice. Postwar liberalism and the remaking of political philosophy. Princeton: Princeton University Press.

Forst, Rainer. 2020. The justification of progress and the progress of justification. In Justification and emancipation. The critical theory of Rainer Forst, 17-37. University Park: Pennsylvania State University Press.

Habermas, Jürgen. 1981. Theorie des kommunikativen Handelns. Frankfurt/Main: Suhrkamp. 2 Bände.

Held, David. 1995. Democracy and the global order. From the modern state to Cosmopolitical governance. Cambridge: Polity Press.

Honneth, Axel. 2011. Das Recht der Freiheit. Grundriss einer demokratischen Sittlichkeit. Berlin: Suhrkamp.

Hont, Istvan. 2005. Jealousy of trade. International competition and the nation-state in historical perspective. Cambridge: Belknap Press of Harvard University Press.

Horkheimer, Max. 1937. Traditionelle und kritische Theorie. Zeitschrift für Sozialforschung 6:245-294.

Jenco, Leigh K., Murad Idris, und Megan C. Thomas (Hrsg.). 2020. The oxford handbook of comparative political theory. Oxford: Oxford University Press.

Kuhn, Thomas. 1962. The structure of scientific revolutions. Chicago: University of Chicago Press.

Latour, Bruno, und Steve Woolgar. 1986. Laboratory life. The construction of scientific facts. Princeton: Princeton University Press.

Mehring, Reinhard. 2019. Ironie der Theorie. Zeitschrift für Politik 66:253-269.

Osrecki, Fran. 2018. Die Geschichte der Gegenwartsdiagnostik in der deutschsprachigen Soziologie. In Geschichte der Soziologie im deutschsprachigen Raum Handbuch Geschichte der deutschsprachigen Soziologie, Bd. 1, Hrsg. Stephan Moebius, Andrea Ploder, 453-475. Wiesbaden: Springer VS.

Salzborn, Samuel (Hrsg.). 2018. Handbuch Politische Ideengeschichte. Zugänge - Methoden - Strömungen. Berlin: Springer.

Slobodian, Quinn. 2018. Globalists. The end of empire and the birth of neoliberalism. Cambridge London: Harvard University Press.

Tyson, Charlie. 2020. The rise of reassurance Lit, The Chronicle of Higher Education. https://www. chronicle.com/article/the-rise- of-reassurance-lit/. Zugegriffen: 28. Febr. 2020.

Weber, Oliver. 2019. Platon wohnt hier nicht mehr. Frankfurter Allgemeine Zeitung. https://blogs.faz.net/ blogseminar/platon-wohnt-hier-nicht-mehr. Zugegriffen: 10. Sept. 2020.

Zürn, Michael. 2004. Regieren jenseits des Nationalstaates. Globalisierung und Denationalisierung als Chance, 2. Aufl., Frankfurt a. M: Suhrkamp. 